\title{
AN APPROXIMATION OF THE ANALYTICAL SOLUTION OF THE FRACTIONAL EULER-LAGRANGE EQUATION
}

\author{
Mariusz Ciesielski ${ }^{1}$, Tomasz Btaszczyk ${ }^{2}$ \\ ${ }^{I}$ Institute of Computer and Information Sciences, Czestochowa University of Technology \\ ${ }^{2}$ Institute of Mathematics, Czestochowa University of Technology \\ Czestochowa, Poland \\ ${ }^{1}$ mariusz.ciesielski@icis.pcz.pl, ${ }^{2}$ tomasz.blaszczyk@im.pcz.pl
}

\begin{abstract}
In this paper the fractional Euler-Lagrange equation of order $\alpha \in(0,1]$ in the finite time interval is considered. This equation is transformed to the integral form by the use of the fractional integral operators. Next, the numerical approximation of the analytical solution is presented. Finally, some examples of numerical solutions are presented.
\end{abstract}

Keywords: fractional Euler-Lagrange equation, numerical solution

\section{Introduction}

The fractional Euler-Lagrange equation (FELE) is an ordinary fractional differential equation with composition of the left and the right derivatives involved. This type of equations is obtained when the minimum action principle and fractional integration by parts rule are applied [1]. Fractional differential equations are in general very difficult to solve (see [2,3] for some solved examples). Moreover, FELE presents an asymmetry: left and right fractional derivatives are involved and it is an additional drawback for the explicit computation of a solution $[4,5]$. Each of these methods leads to a series solution, usually in terms of special functions. On the other hand, computational methods can provide practical approximations of these analytic solutions. Numerous papers have been devoted to the numerical schemes for FELE (see [6-9]). In comparison to our previous works [7, 9], in this paper the solution of FELE deals with the approximation of the analytical solution of FELE based on the numerical evaluation of fractional integrals.

\section{Basic definitions}

In this section, we recall the definitions of the fractional integrals and derivatives. The left and right Riemann-Liouville fractional integrals are defined as follows (see [2] for all the definitions used here): 


$$
\begin{aligned}
& I_{0^{+}}^{\alpha} f(t)=\frac{1}{\Gamma(\alpha)} \int_{0}^{t} \frac{f(\tau)}{(t-\tau)^{1-\alpha}} \mathrm{d} \tau, \quad \text { for } t>0 \\
& I_{b^{-}}^{\alpha} f(t)=\frac{1}{\Gamma(\alpha)} \int_{t}^{b} \frac{f(\tau)}{(\tau-t)^{1-\alpha}} \mathrm{d} \tau, \quad \text { for } t<b
\end{aligned}
$$

where $\Gamma(\cdot)$ denotes the Gamma function and $b \in \square_{+}$. Using (1) and (2) one can define fractional derivatives. For $\alpha \in(0,1]$ the left Riemann-Liouville (3) and the right Caputo (4) derivatives are defined as

$$
\begin{aligned}
& D_{0^{+}}^{\alpha} f(t)=D I_{0^{+}}^{1-\alpha} f(t)=\frac{1}{\Gamma(1-\alpha)} \frac{d}{d t} \int_{0}^{t} \frac{f(\tau)}{(t-\tau)^{\alpha}} \mathrm{d} \tau, \quad \text { for } t>0 \\
& { }^{c} D_{b^{-}}^{\alpha} f(t)=-I_{b^{-}}^{1-\alpha} D f(t)=\frac{-1}{\Gamma(1-\alpha)} \int_{t}^{b} \frac{f^{\prime}(\tau)}{(\tau-t)^{\alpha}} \mathrm{d} \tau, \quad \text { for } t<b
\end{aligned}
$$

\section{Statement of the problem}

Let us consider the following functional $S$ with fixed $\alpha \in(0,1]$ and the parameter $\lambda \in \square$ defined by [5]

$$
S=\int_{0}^{b}\left[\frac{1}{2}\left(D_{0^{+}}^{\alpha} f\right)^{2}-\frac{\lambda}{2} f^{2}\right] d t
$$

Here an unknown function $f$ is absolutely continuous on the interval $[0, b]$. Applying the minimum action principle and fractional integration by parts formula [1] we obtain the following Euler-Lagrange equation

$$
{ }^{c} D_{b^{-}}^{\alpha} D_{0^{+}}^{\alpha} f(t)-\lambda f(t)=0
$$

In this paper we consider Eq. (6) with boundary conditions

$$
f(0)=0, \quad f(b)=L
$$

We can write Eq. (6) in the integral form [5]

$$
f(t)-\lambda I_{0^{+}}^{\alpha} I_{b^{-}}^{\alpha} f(t)=c_{0} t^{\alpha}
$$

where $c_{0}$ is a real constant. Next, by using the Babenko's symbolic calculus method [3] for the composition of the fractional integral operators we have

$$
f(t)=\left(1-\lambda I_{0^{+}}^{\alpha} I_{b^{-}}^{\alpha}\right)^{-1} c_{0} t^{\alpha}
$$


Using the binomial expansion $\left((1-x)^{-1}=\sum_{m=0}^{\infty} x^{m}\right.$, for $\left.|x|<1\right)$ for the operator $\left(1-\lambda I_{0^{+}}^{\alpha} I_{b^{-}}^{\alpha}\right)^{-1}$ we can write solution (9) as

$$
f(t)=c_{0} \sum_{m=0}^{\infty} \lambda^{m}\left(I_{0^{+}}^{\alpha} I_{b^{-}}^{\alpha}\right)^{m} t^{\alpha}=c_{0}\left(t^{\alpha}+\sum_{m=1}^{\infty} \lambda^{m}\left(I_{0^{+}}^{\alpha} I_{b^{-}}^{\alpha}\right)^{m} t^{\alpha}\right)
$$

We have to choose values of parameters $\alpha, \lambda$ and $b$ properly to ensure convergence of the series in (10).

The coefficient $c_{0}$ is determined by the boundary conditions (7)

$$
c_{0}=\frac{L}{\sum_{m=0}^{\infty} \lambda^{m}\left(I_{0^{+}}^{\alpha} I_{b^{-}}^{\alpha}\right)^{m} b^{\alpha}}
$$

Then the analytical solution (10) has the form

$$
f(t)=L \frac{\sum_{m=0}^{\infty} \lambda^{m}\left(I_{0^{+}}^{\alpha} I_{b^{-}}^{\alpha}\right)^{m} t^{\alpha}}{\sum_{m=0}^{\infty} \lambda^{m}\left(I_{0^{+}}^{\alpha} I_{b^{-}}^{\alpha}\right)^{m} b^{\alpha}}
$$

For $\lambda=0$ Eq. (12) simplifies to the form $f(t)=L t^{\alpha} / b^{\alpha}$.

The considered problem in this paper is an estimation of $\left(I_{0^{0}}^{\alpha} I_{b^{-}}^{\alpha}\right)^{m} t^{\alpha}$ in (12) for $m=1, \ldots, \infty$. For $m=1$, the analytical form of $\left(I_{0^{+}}^{\alpha} I_{b^{-}}^{\alpha}\right) t^{\alpha}$ can be expressed by the formula

$$
\left(I_{0^{+}}^{\alpha} I_{b^{-}}^{\alpha}\right) t^{\alpha}=G_{33}^{21}\left[\begin{array}{c}
{[\alpha, 2 \alpha, 3 \alpha+1]} \\
{[3 \alpha, \alpha, 0]}
\end{array} \mid \frac{t}{b}\right]
$$

where $G_{p q}^{k m}$ is the Meijer-G function defined as follows [2]

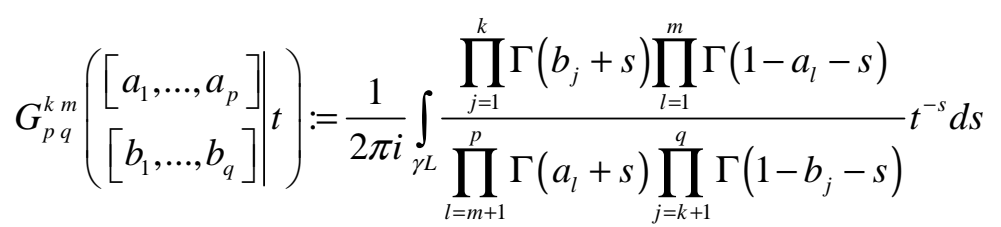


From the numerical point of view approximation of the Meijer-G function is very complicated. For this reason we propose the new numerical method.

\section{Numerical solution}

In order to approximate the analytical solution (12) of (6), we use the homogenous grid of nodes

$$
0=t_{0}<t_{1}<t_{2}<\ldots<t_{i}<t_{i+1}<\ldots<t_{N}=b, \quad t_{i}=i \Delta t, \quad \Delta t=\frac{b}{N}
$$

The value of $f$ at node $t_{i}$ is denoted by $f_{i}=f\left(t_{i}\right), i=0, \ldots, N$.

It will be convenient to introduce the auxiliary functions $g^{m}$

$$
g^{m}(t)=\left(I_{0^{+}}^{\alpha} I_{b^{-}}^{\alpha}\right)^{m} t^{\alpha}, \quad m=0, \ldots, \infty
$$

The following recurrence formula is true

$$
g^{0}(t)=t^{\alpha}, \quad g^{m}(t)=\left(I_{0^{+}}^{\alpha} I_{b^{-}}^{\alpha}\right) g^{m-1}(t), \quad \text { for } m>0
$$

At first we determine numerical schemes for approximation of both fractional operators occurring in Eq. (10). These schemes are based on the trapezoidal rule of integration [9]. The integral (1) vanishes at node $t_{0}\left(\left.I_{0^{+}}^{\alpha} f(t)\right|_{t=t_{0}}=0\right)$, while at nodes $t_{i}, i=1, \ldots, N$, it can be approximated by the formula

$$
\begin{aligned}
\left.I_{0^{+}}^{\alpha} f(t)\right|_{t=t_{i}} & =\frac{1}{\Gamma(\alpha)} \int_{0}^{t_{i}} \frac{f(\tau)}{\left(t_{i}-\tau\right)^{1-\alpha}} d \tau=\frac{1}{\Gamma(\alpha)} \sum_{j=0}^{i-1} \int_{t_{j}}^{t_{j+1}} \frac{f(\tau)}{\left(t_{i}-\tau\right)^{1-\alpha}} d \tau \\
& \approx \frac{1}{\Gamma(\alpha)} \sum_{j=0}^{i-1} \frac{f_{j}+f_{j+1}}{2} \int_{t_{j}}^{t_{j+1}} \frac{1}{\left(t_{i}-\tau\right)^{1-\alpha}} d \tau \\
& =\frac{1}{2 \Gamma(\alpha)} \sum_{j=0}^{i-1}\left(f_{j}+f_{j+1}\right) \int_{j \Delta t}^{(j+1) \Delta t} \frac{1}{(i \Delta t-\tau)^{1-\alpha}} d \tau \\
& =\frac{(\Delta t)^{\alpha}}{2 \Gamma(\alpha+1)} \sum_{j=0}^{i-1}\left(f_{j}+f_{j+1}\right)\left((i-j)^{\alpha}-(i-j-1)^{\alpha}\right) \\
& =\sum_{j=0}^{i} f_{j} w_{i, j}
\end{aligned}
$$


The coefficients $w_{i, j}$ (including the case $i=0$ ) are of the form

$$
w_{i, j}=\frac{(\Delta t)^{\alpha}}{2 \Gamma(\alpha+1)} \begin{cases}0 & \text { for } i=0 \text { and } j=0 \\ i^{\alpha}-(i-1)^{\alpha} & \text { for } i>0 \text { and } j=0 \\ (i-j+1)^{\alpha}-(i-j-1)^{\alpha} & \text { for } i>0 \text { and } 1 \leq j \leq i-1 \\ 1 & \text { for } i>0 \text { and } j=i\end{cases}
$$

Using a similar approach, we determine a discrete form of the integral (2). This operator at node $t_{N}$ has value $\left.I_{b^{-}}^{\alpha} f(t)\right|_{t=t_{N}}=0$, while at the nodes $t_{i}, i=0, \ldots, N-1$ we have

$$
\begin{aligned}
\left.I_{b^{-}}^{\alpha} f(t)\right|_{t=t_{i}} & =\frac{1}{\Gamma(\alpha)} \int_{t_{i}}^{t_{N}} \frac{f(\tau)}{\left(\tau-t_{i}\right)^{1-\alpha}} d \tau=\frac{1}{\Gamma(\alpha)} \sum_{j=i}^{N-1} \int_{t_{j}}^{t_{j+1}} \frac{f(\tau)}{\left(\tau-t_{i}\right)^{1-\alpha}} d \tau \\
& \approx \frac{1}{\Gamma(\alpha)} \sum_{j=i}^{N-1} \frac{f_{j}+f_{j+1}}{2} \int_{t_{j}}^{t_{j+1}} \frac{f(\tau)}{\left(\tau-t_{i}\right)^{1-\alpha}} d \tau \\
& =\frac{1}{2 \Gamma(\alpha)} \sum_{j=i}^{N-1}\left(f_{j}+f_{j+1}\right) \int_{j \Delta t}^{(j+1) \Delta t} \frac{1}{(\tau-i \Delta t)^{1-\alpha}} d \tau \\
& =\frac{(\Delta t)^{\alpha}}{2 \Gamma(\alpha+1)} \sum_{j=i}^{N-1}\left(f_{j}+f_{j+1}\right)\left((j-i+1)^{\alpha}-(j-i)^{\alpha}\right) \\
& =\sum_{j=i}^{N} f_{j} v_{i, j}
\end{aligned}
$$

where the coefficients $v_{i, j}$ (including the case $i=N$ ) look as follows

$$
v_{i, j}=\frac{(\Delta t)^{\alpha}}{2 \Gamma(\alpha+1)} \begin{cases}0 & \text { for } i=N \text { and } j=N \\ (N-i)^{\alpha}-(N-i-1)^{\alpha} & \text { for } i<N \text { and } j=N \\ (j-i+1)^{\alpha}-(j-i-1)^{\alpha} & \text { for } i<N \text { and } i+1 \leq j \leq N-1 \\ 1 & \text { for } i<N \text { and } j=i\end{cases}
$$

From the computational point of view, we propose the following algorithm:

\section{Algorithm 1:}

Input: $\alpha, \lambda, b, L, N, \varepsilon$

Output: $f_{0}, f_{1}, \ldots, f_{N}$ 


$$
\begin{aligned}
& m:=0 \\
& \text { for } i:=0 \text { to } N \text { do } \\
& g_{i}^{m}:=(i b / N)^{\alpha} \\
& f_{i}^{m}:=g_{i}^{m} \\
& \text { repeat } \\
& m:=m+1 \\
& \text { for } i:=0 \text { to } N \text { do } \\
& g_{i}^{\text {temp }}:=\sum_{j=i}^{N} g_{j}^{m-1} v_{i, j} \quad / / v_{i, j} \text { - see Eq. (21) } \\
& \text { for } i:=0 \text { to } N \text { do } \\
& g_{i}^{m}:=\sum_{j=0}^{i} g_{j}^{\text {temp }} w_{i, j} \quad / / w_{i, j} \quad \text { - see Eq. (19) } \\
& \text { for } i:=0 \text { to } N \text { do } \\
& f_{i}^{m}:=f_{i}^{m}+\lambda^{m} g_{i}^{m} \\
& \text { until }\left(\left|\lambda^{m} \max _{i=0, \ldots, N} g_{i}^{m}\right|<\varepsilon\right) \\
& \text { for } i:=0 \text { to } N \text { do } \\
& f_{i}:=L \frac{f_{i}^{m}}{f_{N}^{m}}
\end{aligned}
$$

Array $f^{m}$ stores the partial sum (for indexes from 0 to $m$ ) of the infinite series occurring in (12). Array $g^{\text {temp }}$ is an auxiliary array. In order to reduce the computational memory, one can use only one storage array $g^{m}$ overwritten in every calculation step $m=1,2, \ldots$, in the main loop. Parameter $\varepsilon$ is the threshold to terminate of iteration in the main loop. The running time of the proposed algorithm is $O\left(m_{\max } N\right)$ where $m_{\max }$ is the value of index $m$ when the termination of iteration occurred. The value of $m_{\max }$ depends on the parameters: $\lambda, \alpha, b, N, \varepsilon$.

\section{Results}

Figure 1 presents plots of function (16) for $b=1, m \in\{1,2,5,10\}$ and $\alpha \in\{0.1,0.2,0.4,0.6,0.8,1\}$ on the basis of numerical calulations of Section 3 $(N=1024)$. One can note that if the value of $m$ increases then values of function $g^{m}(t)$ decrease, and for example for $m=100: \max _{0 \leq t \leq 1} g^{100}(t)<9.7 \cdot 10^{-4}$ for $\alpha=0.1$, $\max _{0 \leq t \leq 1} g^{100}(t)<3.1 \cdot 10^{-21}$ for $\alpha=0.6$, and $\max _{0 \leq t \leq 1} g^{100}(t)<4.9 \cdot 10^{-40}$ for $\alpha=1$. 
Comparing plots of $g^{1}(t)$ with plots of function (13) one can see that both plots are the same (with the exceptions of the influence of numerical errors).

Figures 2 and 3 present the numerical evaluation of function (12) for $b=1$, $L=1$, with the parameter $\alpha \in\{0.1,0.2,0.4,0.6,0.8,1\}$ and $\lambda \in\{0.5,1\}$ or $\lambda \in\{-0.5,-1\}$, respectively. Calculations were performed for numerical parameters $N=1024$ and $\varepsilon=10^{-10}$.
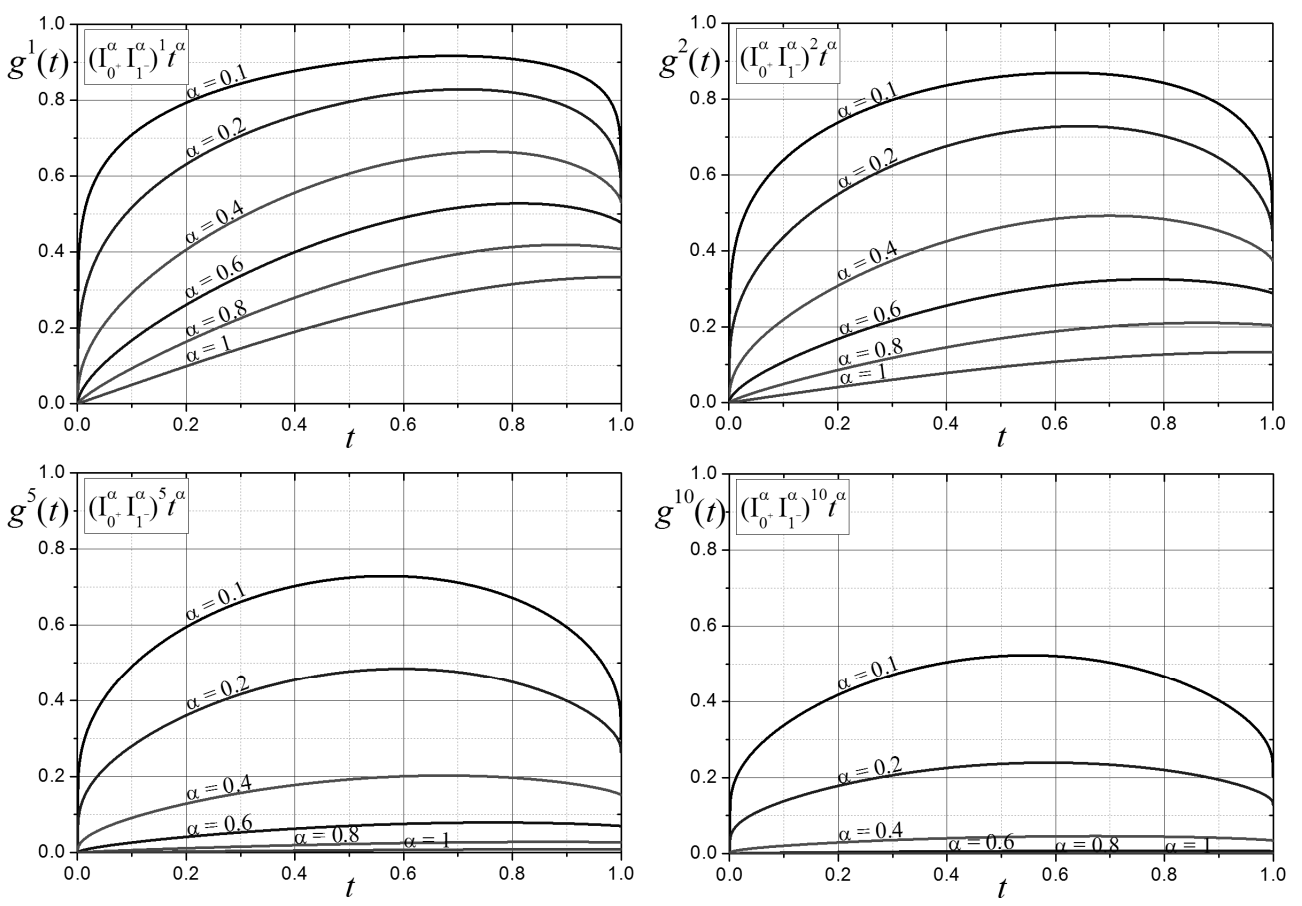

Fig. 1. Numerical approximation of functions $g^{m}(t)$ for $m \in\{1,2,5,10\}, b=1$ and different values of $\alpha$
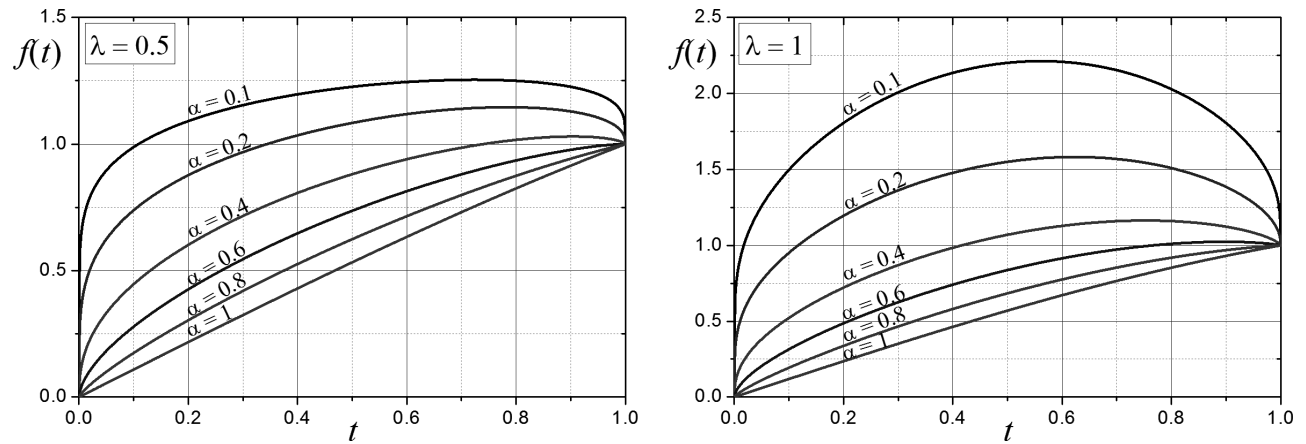

Fig. 2. Numerical solution of Eq. (6) for boundary conditions $f(0)=0, f(1)=1$, and for $\lambda \in\{0.5,1\}$ and different values of $\alpha$ 

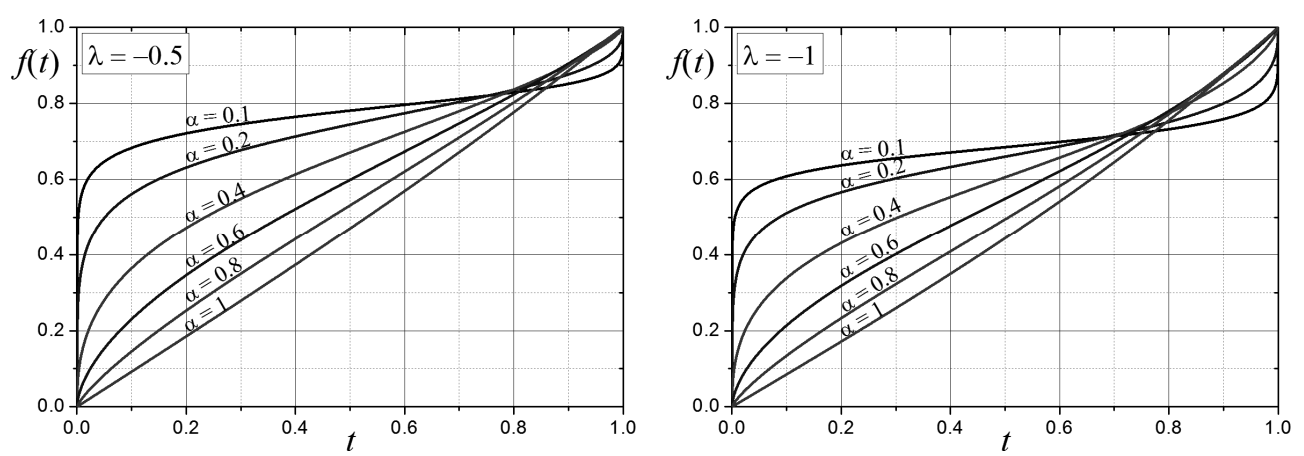

Fig. 3. Numerical solution of Eq. (6) for boundary conditions $f(0)=0, f(1)=1$, and for $\lambda \in\{-0.5,-1\}$ and different values of $\alpha$

\section{Conclusions}

In this paper the numerical algorithm for approximation of the analytical solution of the fractional Euler-Lagrange equation is presented. In comparison with our previous numerical methods [7,9], the present algorithm does not require solving the system of equations. The computational method is relatively fast.

\section{References}

[1] Agrawal O.P., Generalized variational problems and Euler-Lagrange equations, Comput. Math. Appl. 2010, 59, 1852-1864.

[2] Kilbas A.A., Srivastava H.M., Trujillo J.J., Theory and Applications of Fractional Differential Equations, Elsevier, Amsterdam 2006.

[3] Podlubny I., Fractional Differential Equations, Academic Press, San Diego 1999.

[4] Baleanu D., Trujillo J.J., On exact solutions of a class of fractional Euler-Lagrange equations, Nonlinear Dyn. 2008, 52, 331-335.

[5] Klimek M., On Solutions of Linear Fractional Differential Equations of a Variational Type, The Publishing Office of the Czestochowa University of Technology, Czestochowa 2009.

[6] Agrawal O.P., Hasan M.M., Tangpong X.W., A numerical scheme for a class of parametric problem of fractional variational calculus, J. Comput. Nonlinear Dyn. 2012, 7, 021005, 6pp.

[7] Blaszczyk T., Ciesielski M., Klimek M., Leszczynski J., Numerical solution of fractional oscillator equation, Applied Mathematics and Computation 2011, 218, 2480-2488.

[8] Lotfi A., Yousefi S.A., A numerical technique for solving a class of fractional variational problems, Journal of Computational and Applied Mathematics 2013, 237(1), 633-643.

[9] Blaszczyk T., Ciesielski M., Fractional Euler-Lagrange equations - numerical solutions and applications of reflection operator, Scientific Research of the Institute of Mathematics and Computer Science 2010, 2(9), 17-24. 